\title{
IMPLEMENTAÇÃO DE POLÍTICAS PÚBLICAS PARA O ENFRENTAMENTO DE OBESIDADE EM CRIANÇAS E ADOLESCENTES
}

\section{IMPLEMENTATION OF PUBLIC POLICIES FOR COPING WITH OBESITY IN CHILDREN AND ADOLESCENTS}

Silvia Veridiana Zamparoni Victorino', Bianca Machado Cruz Shibukawa', Gabrieli Patricio Rissi", leda Harumi Higarashi'.

'Universidade Estadual de Maringá, Maringá, Paraná, Brasil. *Autor correspondente: Gabrieli Patricio Rissi. Universidade Estadual de Maringá, Maringá (PR), Brasil. Rua dos alecrins, 1141 - Conjunto Habitacional Inocente Vila Nova Júnior. CEP: 87060-100. Telefone: (44) 3259-3533.

E-mail: gabrielirissi@gmail.com.

"Artigo extraído da dissertação "Ações para o enfrentamento da obesidade e sobrepeso em crianças e adolescentes nos municípios da $15^{a}$ Regional de Saúde do Estado do Paraná", apresentada ao Programa de Pós-graduação em Enfermagem da Universidade Estadual de Maringá, em 2014, número de páginas 1-98.

\section{RESUMO}

Objetivou-se analisar a promoção da saúde, enfocando as atividades físicas para crianças e adolescentes para o enfrentamento do sobrepeso e obesidade. Estudo quantitativo, exploratório e transversal, realizado na $15^{a}$ Regional de Saúde do Estado do Paraná. Os dados foram coletados em 2014 por meio de visita aos municípios e consulta aos relatórios públicos do Sistema de Vigilância Alimentar e Nutricional. Observou-se que, na população infanto-juvenil estudada, $40 \%$ apresentaram sobrepeso e/ou obesidade. As ações de Promoção de Saúde implementadas nos municípios variaram de $35 \%$ a $80 \%$ do previsto no Plano de Ações Estratégicas para o Enfrentamento das Doenças Crônicas Não Transmissiveis no Brasil, sendo que a maior parte desenvolve apenas entre 41 e 60\%. Evidenciou-se que cidades com diferentes índices de sobrepeso e obesidade desenvolvem ações semelhantes de promoção em saúde. Portanto, fazem-se necessárias reflexões acerca da participação e atuação dos municípios em busca de soluções para o aumento da qualidade de vida infanto-juvenil.

Palavras-chaves: promoção da saúde; obesidade pediátrica; enfermagem; saúde da criança.

\section{ABSTRACT}

The study aimed to analyze health promotion, focusing on physical activities for children and adolescents to cope with overweight and obesity. This is a quantitative, exploratory and crosssectional study, carried out in the $15^{\text {th }}$ Health Region of the State of Paraná. Data were collected in 2014 through visits to the municipalities and consultation of the public reports from the Food and Nutrition Surveillance System. The results show that $40 \%$ of the children and adolescents studied were overweight and/or obese. The Health Promotion actions implemented in the municipalities ranged from $35 \%$ to $80 \%$ of what is predicted in the Strategic Action Plan for Coping with Chronic Noncommunicable Diseases in Brazil, with the majority developing only between 41 and $60 \%$. Cities with different rates of overweight and obesity develop similar actions of health promotion. Therefore, there is need to reflect on the participation and actions of the municipalities seeking solutions to increase the quality of life of children and adolescents.

Keywords: health promotion; pedriatric obesity; nursing; child health. 


\section{INTRODUÇÃO}

A obesidade é uma doença que se manifesta por meio do armazenamento de gordura no organismo, o que pode ocasionar complicações metabólicas. Pode ser definida ainda como uma doença decorrente de um processo desequilibrado entre gasto e suprimento energético, resultando em ganho de peso, podendo se manifestar nos indivíduos de qualquer faixa etária, etnia ou situação socioeconômica ${ }^{(1-2)}$.

A população mundial está enfrentando uma epidemia de sobrepeso e obesidade, fato este expressado pela triplicação de população obesa de 1975 a 2016. Estima-se que cerca de 381 milhões de crianças e adolescentes de 0 a 19 anos estejam com sobrepeso ou obesidade ${ }^{(3)}$.

No Brasil, seguindo-se a tendência apontada por outros países, também é possível observar as taxas de sobrepeso e obesidade crescendo exponencialmente, sendo o sexo feminino o mais afetado. Tal aumento sugere ainda a insuficiência ou ineficiência de políticas públicas voltadas para este $\operatorname{agravo}^{(4)}$.

Outro fator que contribui com o aumento do sobrepeso e obesidade na população vigente consiste no próprio processo de urbanização aliado ao estilo de vida adotado. As brincadeiras tornam-se cada vez mais tecnológicas, restringindo ou anulando as possibilidades de movimento, além da modificação dietética, onde os alimentos ultra processados estão cada vez mais presentes no cotidiano familiar, o que contribui com o desenvolvimento de uma dieta obesogênica, podendo implicar em riscos à saúde ${ }^{(5)}$.

Desta forma, a obesidade é reconhecida como um grave problema de saúde pública. Portanto, como medida de enfrentamento a este agravo, o Ministério da Saúde lançou, em 2011, o Plano de Ações Estratégicas para o Enfrentamento das Doenças Crônicas Não Transmissíveis no Brasil, objetivando a promoção, o desenvolvimento e a implementação de políticas públicas efetivas e integradas, visando a reduzir a prevalência de obesidade, com ênfase às atividades físicas, alimentação saudável, combate ao tabagismo e uso de álcool (5-6).

Neste contexto, o objetivo do estudo foi analisar a promoção da saúde, enfocando as atividades físicas e práticas corporais para crianças e adolescentes implementadas nos municípios da $15^{\mathrm{a}}$ Regional de Saúde do Estado do Paraná para o enfrentamento do sobrepeso e obesidade.

\section{MATERIAL E MÉTODO}

Estudo descritivo de recorte transversal e abordagem quantitativa, realizado no âmbito da $15^{\mathrm{a}}$ Regional de Saúde do estado do Paraná, a qual engloba 30 municípios da região noroeste do estado, apresentando um total de 724.215 habitantes ${ }^{(7)}$.

A pesquisa foi inicialmente submetida à apreciação pela direção da $15^{\mathrm{a}}$ Regional de Saúde do Estado do Paraná, obtendo-se a adesão de 100\% dos municípios ao estudo. Realizou-se uma reunião prévia com cada gestor municipal, visando a viabilizar as visitas aos municípios para a realização de entrevistas.

A coleta de dados ocorreu nos meses de abril a junho de 2014, por meio da implementação de duas etapas, as quais consistiram no levantamento de dados secundários para caracterização das ações de promoção à saúde efetivadas pelos municípios para o controle do sobrepeso e obesidade infanto-juvenil e nas entrevistas com os gestores ou informantes indicados pelos mesmos, abordando a temática central do estudo. 
Para o levantamento dos dados secundários, realizou-se consulta ao Sistema de Vigilância Alimentar e Nutricional, o que permitiu consultar os relatórios de crianças e adolescentes em fase escolar, na faixa etária de 5 a 19 anos, quanto ao estado nutricional: sobrepeso, obesidade e obesidade grave, referentes ao período entre 2006 a 2013.

Para as entrevistas com os gestores, foi utilizado roteiro estruturado, dividido em duas partes, envolvendo aspectos da caracterização da amostra e da temática central do estudo, com o intuito de levantar informações quanto às ações de promoção de saúde e de atividades físicas oferecidas pelos municípios, baseadas nas recomendações do Plano de Ações Estratégicas para o Enfrentamento das Doenças Crônicas Não Transmissíveis referentes ao período de 2011 a 2022.

As informações foram organizadas em planilhas, por meio do Programa Microsoft Excel, versão 2016. Inicialmente, realizou-se análise descritiva e exploratória dos dados coletados a partir das entrevistas, os quais foram organizados em tabelas. Todas as análises estatísticas foram conduzidas com o Programa Linguagem R, versão 3.5.1.

As ações desenvolvidas pelos municípios foram categorizadas em três grupos de acordo com o percentual de ações implantadas em relação ao rol preconizado pelo Plano de Ações Estratégicas para o Enfrentamento das Doenças Crônicas Não Transmissíveis, sendo eles: Grupo I (GI) - composto por municípios que apresentaram 35\% a 50\%; Grupo II (GII) - composto por municípios onde este percentual variou de $51 \%$ a $65 \%$ e Grupo III (GIII) composto por municípios com $66 \%$ a $80 \%$ de ações implantadas.

O estudo respeitou os aspectos éticos contidos nas Resoluções 466/2012(8) e 510/2016 ${ }^{(9)}$ do Conselho Nacional de Saúde. O projeto foi aprovado pelo Comitê Permanente de Ética em Pesquisa Envolvendo Seres Humanos da Universidade Estadual de Maringá, conforme parecer 623.548/2014. Todos os direitos relativos à livre participação e ao anonimato foram garantidos aos entrevistados, que registraram sua anuência por meio da assinatura do Termo de Consentimento Livre e Esclarecido.

\section{RESULTADOS}

Dos 30 municípios participantes, 56\% possuíam apenas uma equipe de Estratégia Saúde da Família (ESF), 22\% apresentavam entre três e oito equipes, 16\% contavam com duas equipes e 3\% dispunham de 66 equipes. Em relação ao total de Unidade Básica de Saúde (UBS), 50\% apresentavam uma unidade para todo o município, $25 \%$ possuíam de cinco a onze unidades, $20 \%$ possuíam duas e $3 \%$ contava com uma rede formada por 29 UBS.

Em relação aos gestores municipais entrevistados, em 27\% dos casos, foram representados pelos próprios Secretários de Saúde. O profissional mais indicado pelos gestores para a prestação de informações e realização de entrevistas foram os enfermeiros, correspondendo a 53\% das indicações. As porcentagens de adesão as ações de promoções à saúde, voltadas para o enfrentamento do sobrepeso e obesidade, estão dispostas na Tabela 1. 
Tabela 1 - Percentagem de ações de Promoção de Saúde desenvolvidas pelos municípios, 2014.

\begin{tabular}{l|l|l|l|l|l} 
Grupo & $\begin{array}{l}\text { \% ações de } \\
\text { promoção a } \\
\text { saúde }\end{array}$ & $\begin{array}{l}\mathbf{n}^{\circ} \text { de } \\
\text { municípios }\end{array}$ & $\begin{array}{l}\text { \% de } \\
\text { municípios }\end{array}$ & $\begin{array}{l}\text { Mediana de sobrepeso/ } \\
\text { obesidade - Crianças }\end{array}$ & $\begin{array}{l}\text { Mediana de sobrepeso/ } \\
\text { obesidade - Adolescentes }\end{array}$ \\
\hline GI & $35-50$ & 17 & 56,6 & $25(0,00 ; 40,00)$ & $0(0,00 ; 41,22)$ \\
\hline GII & $51-65$ & 8 & 26,6 & $34,95(15,60 ; 47,22)$ & $27,3(0,00 ; 43,78)$ \\
\hline GIII & $66-80$ & 5 & 16,6 & $33,5(22,70 ; 43,60)$ & $30,7(0,00 ; 41,70)$ \\
\hline
\end{tabular}

A quantidade de ações de promoção em saúde implementadas pela totalidade dos municípios analisados e relacionadas à atividade física variou de $35 \%$ a $80 \%$, sendo que a maioria deles $(20$ municípios) desenvolvia entre 41\% e 60\% das ações preconizadas pelo Plano de Ações Estratégicas para o Enfrentamento das Doenças Crônicas Não Transmissíveis, sendo que apenas seis municípios desenvolviam de 61 a $80 \%$. Destaca-se ainda que nenhum dos municípios desenvolvia mais que $80 \%$ das ações preconizadas.

É importante ressaltar que o GI, o qual aglutina os municípios com menor percentual de ações implantadas, apresentou também a menor mediana de sobrepeso e obesidade em crianças e adolescentes. O GII, por sua vez, obteve a maior mediana da prevalência de sobrepeso em crianças; já o GIII revelou a maior mediana de prevalência em adolescentes. A adesão dos municípios às ações propostas pela Plano de Ações Estratégicas para o Enfrentamento das Doenças Crônicas Não Transmissíveis está representada na Tabela 2.

Tabela 2 - Percentagem de adesão dos municípios às ações propostas pelo Plano de Ações Estratégicas para o Enfrentamento das Doenças Crônicas Não Transmissíveis, 2014.

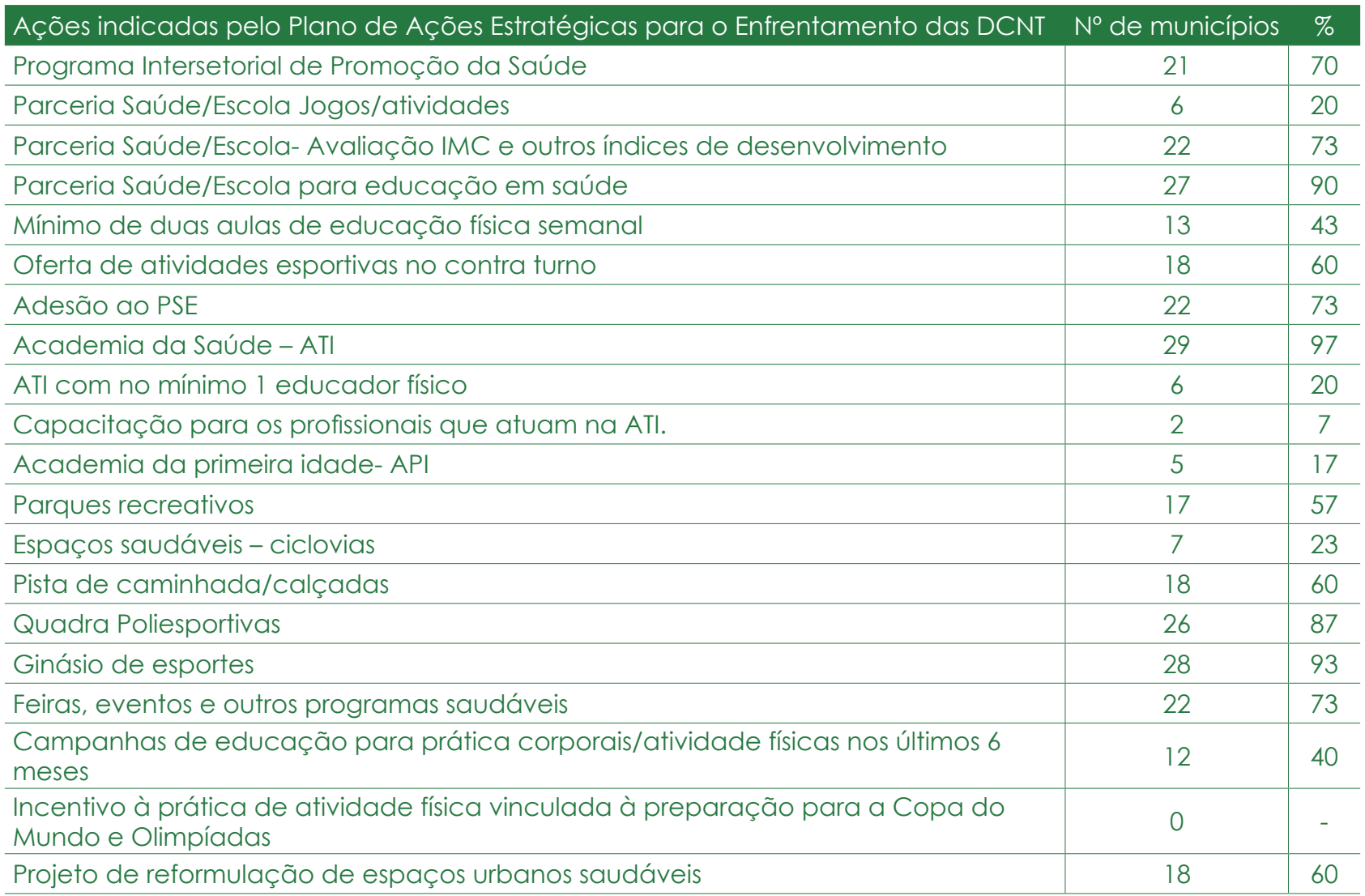


Para estabelecer a rede de associação entre a prevalência de sobrepeso e obesidade com o percentual de ações implementadas pelos municípios, elegeu-se o ano de 2013 para retratar a prevalência atual. Como limite de classificação do SP/OB, foi adotada a mediana de prevalência encontrada no conjunto dos municípios investigados como forma de agregar os municípios com maior e menor número de ações. A opção pela utilização da mediana deu-se em função da inexistência de um indicador nacional da prevalência deste agravo, de maneira que a mediana permitiria dicotomizar os municípios em relação a um marcador central de distribuição dos dados.

Os municípios que apresentaram índices de sobrepeso e obesidade em crianças abaixo da mediana foram Anguio, Uniflor, Nova Esperança, Paiçandu, Munhoz de Melo, Itambé, Ourizona, Paranacity, Atalaia, Floraí, Marialva, Colorado, Lobato, Santo Inácio e Presidente Castelo Branco. Já os municípios pertencentes à $15^{a}$ Regional de Saúde que apresentaram índices acima da mediana foram Astorga, Ivatuba, Floresta, Doutor Camargo, Iguaraçu, Maringá, Mandaguaçu, Flórida, Sarandi, São Jorge do Ivaí, Itaguaji, Santa Fé, Santa Inês, Nossa Senhora das Graças e Mandaguari.

Em relação aos municípios que apresentaram índices de sobrepeso e obesidade em adolescentes abaixo da mediana, foram Angulo, Uniflor, Paiçandu, Itambé, Ourizona, Paranacity, Atalaia, Santa Fé, Floraí, Colorado, Lobato, Santo Inácio, Senhora das Graças, Mandaguari e Presidente Castelo Branco. Já os municípios que apresentaram índices acima da mediana foram Astorga, Ivatuba, Floresta, Nova Esperança, Doutor Camargo, Iguaraçu, Munhoz de Melo, Maringá, Mandaguaçu, Marialva, Flórida, Sarandi, São Jorge do Ivaí, Itaguaji e Santa Inês.

Destaca-se que em ambas classificações e faixas etárias, as quantidades de ações de promoção da saúde desenvolvidas tiveram uma importante variação. Ademais, evidenciou-se uma acentuada semelhança entre municípios de diferentes grupos, de tal forma que municípios com diferentes índices de sobrepeso e obesidade em crianças e adolescentes demonstraram desenvolver ações similares de promoção em saúde.

\section{DISCUSSÃO}

A caracterização dos entrevistados evidenciou o papel de destaque ocupado pelo enfermeiro no que concerne ao desenvolvimento de ações de promoção em saúde, sendo os profissionais mais indicados pelos secretários municipais para a prestação das informações atinentes à pesquisa, no que tange às ações e à estrutura municipal disponíveis para o enfrentamento da obesidade e sobrepeso infantil.

Tal achado ratifica a importância deste profissional, principalmente em função da proximidade do cuidado em enfermagem com a promoção da vida. Nesse sentido, o trabalho do enfermeiro pautado no processo de cuidado constitui-se em base para a prática da promoção global da saúde ${ }^{(10)}$.

No que concerne à distribuição de sobrepeso e obesidade em crianças e adolescentes encontrada a partir da consulta aos dados do Sistema de Vigilância Alimentar e Nutricional, verificou-se o crescimento acelerado nos índices deste agravo nos últimos cinco anos, de tal modo que a prevalência identificada em 2013 foi bastante similar à nacional. Tais achados corroboram a literatura encontrada, a qual aponta alta prevalência de excesso de peso e obesidade entre crianças e adolescentes em todas as regiões brasileiras ${ }^{(11)}$.

Não obstante a esta tendência de evolução do agravo, há de se considerar que, na fase inicial de utilização do Sistema de Vigilância Alimentar e Nutricional, podem ter ocorrido falhas na alimentação 
dos dados, que foi sendo aprimorada no decorrer dos anos, inviabilizando um diagnóstico fiel acerca do processo de evolução do SP/OB nestas faixas etárias ${ }^{(12)}$.

Com relação à análise da implantação de ações de promoção em saúde voltadas à atividade física e ao controle do excesso de peso, observou-se que a maioria desenvolve até $60 \%$ das ações preconizadas. Tais resultados revelam a falta de priorização de investimentos nestas ações, contrariando o que seria esperado e necessário, especialmente devido aos crescentes índices de sedentarismo encontrados nesta população.

Os dados da Pesquisa Nacional de Saúde do Escolar (PeNSE) revelaram que apenas 30\% dos escolares são ativos, enquanto 63\% encontram-se em situação insuficientemente ativa e quase $7 \%$ estão inativos. A PeNSE constatou ainda que o hábito de assistir a duas horas ou mais de televisão, em pelo menos um dia da semana, está presente em $78 \%$ dos estudantes investigados ${ }^{(6,13)}$.

Neste contexto, evidencia-se a necessidade de trazer à pauta de discussões estratégias que busquem garantir estrutura para a prática regular de atividades físicas na infância e adolescência. Tais ações devem prever o acesso a estes benefícios por todas as classes sociais, garantindo a estrutura física e segurança que permitam a todos os cidadãos alternativas para o desenvolvimento de hábitos saudáveis ${ }^{(14)}$.

A literatura aponta que o acesso às áreas públicas de lazer interfere na prática de atividade física. Um estudo, realizado na cidade do Rio de Janeiro, concluiu que a prática de atividade física de lazer variou significativamente conforme os bairros de residência, sendo mais prevalente entre residentes de bairros com melhores condições sociais e de acesso a áreas públicas de lazer, independentemente das características individuais ${ }^{(15)}$.

Um aspecto estrutural observado foi a ausência de Academias para a Primeira Idade (API), contrariando a necessidade imposta pelos indicadores atuais de sobrepeso e obesidade infantil. Este achado pode denotar a falta de um olhar mais atento por parte das gestões municipais, no que tange à problemática crescente que este agravo vem representando para a sociedade.

Outro fator a ser considerado que pode interferir na oferta de ações voltadas aos adolescentes é a dificuldade que os enfermeiros e equipe da atenção básica apresentam para trabalhar com este público, o que conduz, invariavelmente, à proposição e desenvolvimento de ações esporádicas e pontuais, e não de forma efetiva e contínua ${ }^{(16)}$.

No tocante às ações intersetoriais de promoção em saúde, destacaram-se as parcerias firmadas entre a Secretaria de Educação e a Secretaria de Saúde, visando ao desenvolvimento de ações de Educação em Saúde. Estes resultados refletem a visão dos profissionais de saúde, os quais percebem, no ambiente escolar, um espaço social que contribui para a promoção da saúde, já que é onde são formadas as opiniões dos educandos e de seus familiares ${ }^{(17)}$.

No entanto, as intervenções desenvolvidas nas escolas ainda se mostram muito pontuais, contrariando as recomendações expressas nos parâmetros curriculares nacionais, as quais estabelecem que os conteúdos de saúde devem estar inseridos no conteúdo curricular, por meio de abordagem transversal e interdisciplinar. Desta forma, a educação em saúde na escola, embora de extrema importância, tem sido pouco efetiva em sua prática, não conseguindo provocar mudanças de atitudes que conduzam a opções mais saudáveis de vida ${ }^{(18)}$.

Dentro da perspectiva de ampliação das ações de saúde no ambiente escolar, o Programa Saúde na Escola (PSE) firmou-se como uma ferramenta desencadeadora de ações intersetoriais, atingindo 
o objetivo de promover a saúde e a cultura da paz, reforçando a prevenção de agravos à saúde, bem como fortalecer a relação entre as redes públicas de saúde e de educação, proporcionando contato permanente das equipes de ESF para avaliação das condições de saúde dos educandos, para que possa ser realizado acompanhamento das necessidades ${ }^{(19)}$.

Ainda com relação às ações implementadas no âmbito escolar, detectou-se um baixo índice de atividades físicas, mesmo estas sendo consideradas um elemento importante para a formação educacional, podendo auxiliar na detecção de fatores de risco à saúde, levando à melhor qualidade de vida na fase adulta ${ }^{(14,20)}$.

Verificou-se uma grande variação de ações de promoção em saúde nos municípios, o que permite refletir acerca do processo de planejamento e implementação destas ações, as quais frequentemente acontecem de forma aleatória, sem critérios científicos ou um diagnóstico sistemático prévio das realidades locais.

Sem o embasamento epidemiológico, inviabiliza-se a implantação de Políticas de Promoção de Saúde efetivas, ficando a população à mercê de ações esporádicas sem impacto na qualidade de vida, visto que o propósito da vigilância em saúde é fornecer orientação técnica para o controle dos agravos e danos, contribuindo para a identificação de prioridades e avaliação dos serviços de saúde (21).

Os limites do estudo encontram-se na manutenção inadequada de dados do Sistema de Vigilância Alimentar e Nutricional em seus primeiros anos, seja pelo curto espaço de tempo transcorrido desde a implantação do Plano de Ações Estratégicas para o Enfrentamento das Doenças Crônicas Não Transmissíveis, inviabilizando uma avaliação completa de sua efetividade e de seus impactos, os quais conduzem à necessidade de novos estudos sobre o tema no sentido de avaliar os efeitos destas proposições a médio e a longo prazo.

\section{CONCLUSÕES}

Apesar dos altos índices de sobrepeso e obesidade infantil encontrados no presente estudo e, não obstante, a existência de inúmeras propostas em nível mundial e nacional para enfrentamento desta problemática, foi possível verificar que as gestões municipais pouco têm avançado no sentido da criação de ambientes mais saudáveis e de estratégias de promoção em saúde capazes de controlar este agravo na sociedade.

As ações voltadas para práticas esportivas ainda se mostram escassas frente às reais necessidades desta clientela. Dentre as ações intersetoriais, destaca-se a parceria exitosa entre as áreas de saúde e educação, efetivada por meio do Programa Saúde na Escola, ainda que necessite de incremento para se estabelecer como uma política transversal e permanente.

Faz-se necessário reforçar a interdisciplinaridade, reconhecendo os obstáculos estruturais, humanos e logísticos que ainda persistem no contexto da promoção em saúde junto à clientela infanto-juvenil como pontos de partida para a busca de soluções a um estilo de vida mais saudável, com vistas à maior qualidade de vida futura.

\section{REFERÊNCIAS}

1-PAES, S. T.; MARINS, J. C. B.; ANDREAZZI, A. Efeitos metabólicos do exercício físico na obesidade infantil: uma visão atual. Revista Paulista de Pediatria, São Paulo, v.33, n.1, p.122-129, 2015. 
2- DIAS, P. C.; HENRIQUES, P.; ANJOS, L. A.; BURLANDY, L. Obesity and public policies: the Brazilian government's definitions and strategies. Cadernos de Saúde Pública, Rio de Janeiro, v.33, n.7, 2017.

3- WORLD HEALTH ORGANIZATION. Obesity and overweight. 2016 (online). Disponível em: http:// www.who.int/en/news-room/fact-sheets/detail/obesity-and-overweight. Acesso em: 15 de Agosto de 2018.

4- JARDIM, J. B.; SOUZA, I. L. Childhood obesity in Brazil: an integrative review. Journal of Management Primary Health Care, São Paulo, v.8, n.1, p. 66-90, 2017.

5- SARAIVA, N. C. G.; MEDEIROS, C. C. M.; ARAUJO, T. L. Serial album validation for promotion of infant body weight control. Revista Latino-Americana de Enfermagem, Ribeirão Preto, v.26, e2998, 2018.

6- BRASIL. Ministério da Saúde. Secretaria de Vigilância em Saúde. Departamento de Análise da Situação em Saúde. Coordenação Geral de Doenças e Agravos Não Transmissíveis. Plano de Ações Estratégicas para o Enfrentamento das Doenças Crônicas Não Transmissíveis no Brasil, 2011 2022; 2011.

7- BRASIL. Instituto Brasileiro de Geografia e Estatística. Sinopse do Censo Demográfico 2010. Ministério do Planejamento, Orçamento e Gestão. Rio de Janeiro/RJ: IBGE; 2010.

8- BRASIL. Ministério da Saúde. Conselho Nacional de Saúde. Resolução n 466, de 12 de dezembro de 2012. Diário Oficial [da] República Federativa do Brasil, Brasília, DF, 12 dez. 2012. Disponível em: http://bvsms.saude.gov.br/bvs/saudelegis/cns/2013/res0466_12_12_2012.html

9- BRASIL. Ministério da Saúde. Conselho Nacional de Saúde. Resolução n 510, de 7 de abril de 2016. Diário Oficial [da] República Federativa do Brasil, Brasília, DF, 24 maio 2016. Seção 1. p. 44-46. Disponível em: http://conselho.saude.gov.br/resolucoes/2016/ Reso510.pdf

10- NASCIMENTO, M. G. G.; NADALETI, N. P.; VILELA, S. C.; TERRA, F. S.; SILVA, S. A.; RESCK, Z. M. R. O processo de trabalho do enfermeiro na promoção da saúde mental da população atendida na ESF: uma análise reflexiva. Revista de Enfermagem do Centro-Oeste Mineiro, Minas Gerais, v.7, e2097, 2017.

11 - NIEHEUS, J. R.; GONZALES, A. L.; LEMOS, R. R.; BEZERRA, P. P.; HAAS, P. Prevalence of Overweight and Obesity in Children and Adolescents from the Age Range of 2 to 19 Years Old. International Journal of Pediatrics, Irã, 2014:583207, 2014.

12- NASCIMENTO, F. A.; SILVA, A. S.; JAIME, P. C. Coverage of assessment of nutritional status in the Brazilian Food and Nutritional Surveillance System, 2008-2013. Cadernos de Saúde Pública, Rio de Janeiro, v.33, n.12, e00161516, 2017.

13- BRASIL. Ministério da Saúde. Secretaria de Vigilância em Saúde. Departamento de Análise de Situação de Saúde. Pesquisa Nacional de Saúde do Escolar. Brasília: Ministério da Saúde; 2012.

14- FREIRE, F. S.; LÉLIS, F. L. O.; FONSECA FILHO, J. Á.; NEPOMUCENO, M. O.; SILVEIRA, M. F. Regular physical activity: a population-based study in north Minas Gerais, Brazil. Revista Brasileira de Medicina do Esporte, São Paulo, v. 20, n. 5, 2014.

15- BOCLIN, K. L. S.; FAERSTEIN, E.; LEON, A. C. M. P. Características contextuais de vizinhança e atividade física de lazer: Estudo Pró-Saúde. Revista de Saúde Pública, São Paulo, v.48, n.2, P. 249$57,2014$. 
16- ARAÚJO, M. S.; SALES, L. K. O.; ARAÚJO, M. G.; MORAIS, I. F.; MORAIS, F. R. R.; VALENÇA, C. N. Difficulties faced by nurses to develop direct actions to adolescents in primary care. Revista de enfermagem UFPE on line, Pernambuco, v.10, n.5, p. 4219-25 2016.

17- SOUZA, K. R. S. Representações sociais de professores da educação profissional em saúde sobre o ser professor. Natal, 2018. 255 p. Dissertação (Mestrado em Educação) - Centro de Educação, Universidade Federal do Rio Grande do Norte.

18- NEIRA, M. G.; BORGES, C. C. O. Esquadrinhar e Governar: análise das recomendações do CONFEF para a Educação Física escolar. Educação e Realidade, Porto Alegre, v.43, n.2, p. 571-590, 2018.

19 - BRASIL. Ministério da Saúde. Secretaria de Atenção à Saúde. Departamento de Atenção Básica. Instrutivo PSE. Brasília: Ministério da Saúde, 2011.

20- SANTOS, J. P. P.; PRATI, A. R. C.; MOLENA, C. A. F. Associação entre pressão arterial e indicadores de obesidade geral e central em escolares: pistas para cuidar-educar da criança na escola. Ciência, Cuidado e Saúde, Maringá, v.12, n.1, p. 146-54, 2013.

21- FERREIRA, J.; CELUPPI, I. C.; BASEGGIO, L.; SAVI, D.; FAGANELLO, V. S.; SOUZA, J. B. Regional planning of health services: what do the managers say? Saúde e Sociedade, São Paulo, v.27, n. 1, p. 69-79, 2018. 